\title{
Radiation recall pneumonitis induced by ifosfamide for malignant soft tissue tumor arising from the chest wall: A case report
}

\author{
HIROAKI MURATA, KENZOU KOYAMA, YASUNOBU TAKEZAWA and YASUNORI NISHIGAKI \\ Department Orthopaedics, Matsushita Memorial Hospital, Moriguchi, Osaka 570-8540, Japan
}

Received November 3, 2017; Accepted December 28, 2017

DOI: $10.3892 /$ mco.2018.1562

\begin{abstract}
The present study reports a rare case of radiation recall pneumonitis attributed to ifosfamide that was used as a single antitumor agent for a recurrent malignant soft tissue tumor, arising from the chest wall. A 74-year-old man, who had a mass in his right upper chest wall, underwent surgical excision under the diagnosis of chronic expanding hematoma. He was referred to the present hospital with a recurrent tumor as the excised tumor was diagnosed as undifferentiated high-grade sarcoma. The extension of the tumor following previous surgery was aggressive and the tumor margins were unclear on magnetic resonance imaging. Wide excision was done with adequate margins for the new recurrent tumor as it was difficult to set sufficient tumor margins following the previous surgery. Radiotherapy (60 Gy) on his right subclavicular area and chemotherapy with ifosfamide ( $3.5 \mathrm{~g} \mathrm{x} 4$ days/cycle) was started 1 month following surgery to control the growth of residual tumors and to prevent metastases. The third cycle of chemotherapy with ifosfamide ( $3 \mathrm{~g} \mathrm{x} 4$ days) was started 80 days following the final surgery and 15 days following termination of radiotherapy. Following administration, he felt short of breath and dyspnea gradually increased. This condition was diagnosed as interstitial pneumonitis induced by ifosfamide following radiotherapy. Although corticosteroid pulse therapy was performed, he died due to respiratory failure. To the best of the author's knowledge, this is the first case report of radiation recall pneumonitis induced by ifosfamide used as a single anti-tumor agent. Caution is needed when ifosfamide is planned for patients who have undergone previous radiotherapy to the chest.
\end{abstract}

\footnotetext{
Correspondence to: Dr Hiroaki Murata, Department Orthopaedics, Matsushita Memorial Hospital, 5-55 Sotojima-cho, Moriguchi, Osaka 570-8540, Japan

E-mail: murah@koto.kpu-m.ac.jp
}

Key words: ifosfamide, radiotherapy, malignant soft tissue tumor, radiation recall pneumonitis, chest wall

\section{Introduction}

Excision with adequate margins for malignant soft tissue tumors arising from the chest wall is often challenging. Moreover, treatment after recurrence or inadequate excision is more difficult because of unexpected margins. The prognosis of malignant soft tissue tumors arising from the chest wall is poor compared with tumors arising from the extremities. Radiotherapy is additionally required in such difficult cases and chemotherapy is performed to prevent local recurrence or metastases.

When these adjuvant therapies are performed for tumors arising from the chest wall, interstitial pneumonitis must be considered as a complication. In particular, radiation recall pneumonitis, a rare inflammatory reaction in the previously irradiated lung field caused by administration of antitumor agents, should be kept in mind (1).

Here, we present a case of interstitial pneumonitis induced by ifosfamide following radiotherapy after excision of a recurrent malignant soft tissue tumor arising from the chest wall. To our knowledge, this is the first report of radiation recall pneumonitis attributable to ifosfamide used as a single antitumor agent. Written informed consent was obtained from the patient.

\section{Case report}

A 74-year-old man visited his doctor with a 2-month history of a painless mass in his right upper chest wall after contusion. He was diagnosed with hematoma and underwent curettage. The mass rapidly increased in size 2 months after the previous surgery (Fig. 1), and surgical excision was performed under the diagnosis of chronic expanding hematoma. He was then referred to our hospital 1 month after the last surgery because the mass was histologically diagnosed as undifferentiated high grade sarcoma. He had a new growing mass on the lateral edge of the previous skin incision (Fig. 2). The extension of the tumor after surgery was aggressive and was unclear on MR imaging (Fig. 3). It was difficult to set sufficient tumor margins. He had good general condition for his age. Past and family histories were unremarkable. He had no metastasis. Due to the extent of the disease, he was not considered to be a candidate for additional curative surgical excision including the previous lesion. Therefore, we planned wide excision for the new tumor, and adjuvant radiotherapy and chemotherapy after surgery 


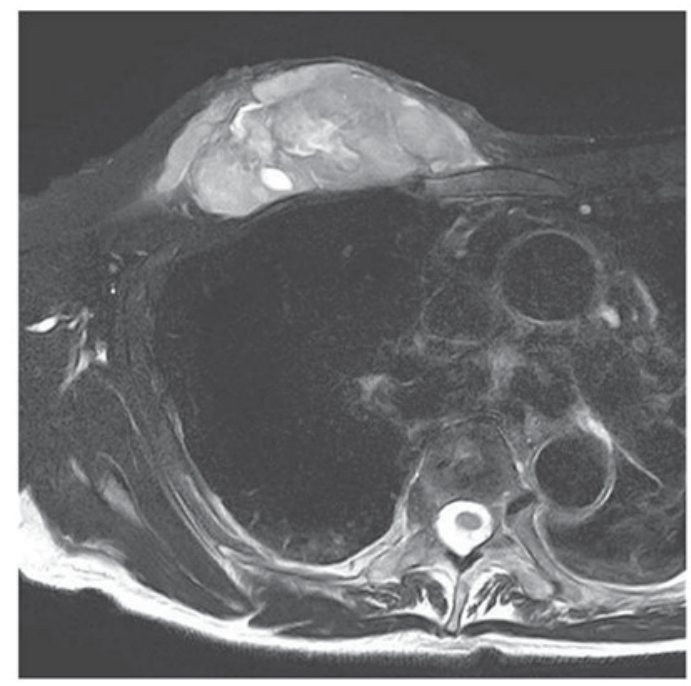

Figure 1. T2-weighted MR image demonstrated the heterogeneous lesion with hyper intensity in the muscle.

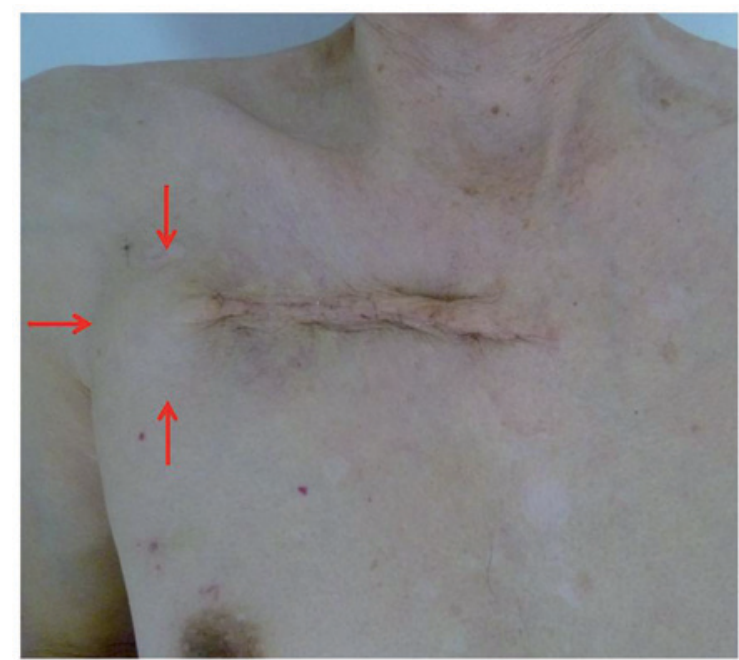

Figure 2. There was an $11-\mathrm{cm}$ incision line and $4-\mathrm{cm}$ diameter mass on the lateral side (arrow).

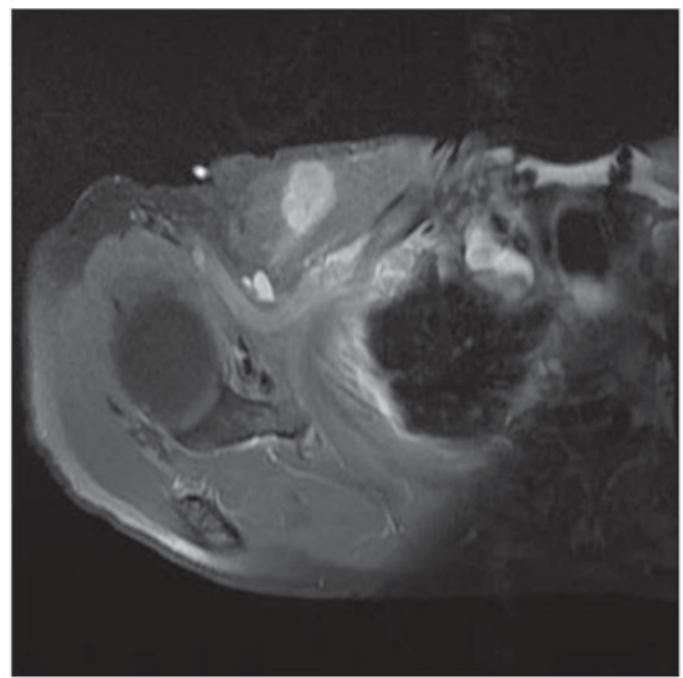

Figure 3. The tumor was located in the pectoralis major. In images obtained by gadolinium-enhanced MRI, a part of the tumor margin was unclear.

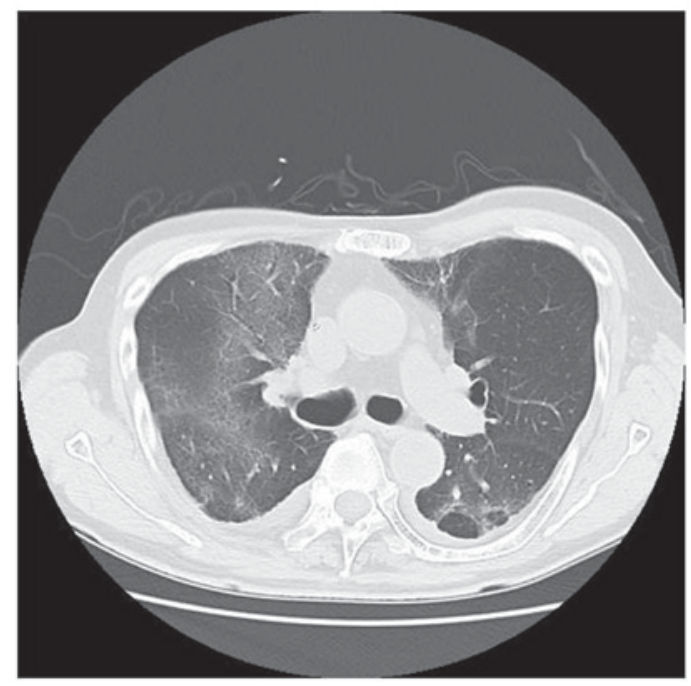

Figure 4. Computed tomography images revealed a ground-glass appearance in the pulmonary lobe.

because of the high risk of local recurrence and metastasis. After wide excision was performed with adequate margins for the new tumor, concurrent chemoradiotherapy with ifosfamide and local radiotherapy up to 60 Gy in 30 fractions of 2 Gy over 6 weeks was started 1 month after the final surgery to control the growth of any residual tumors. The irradiated region around the right subclavicular area was set to not include lung as much as possible. Although our protocol for this tumor was ordinary combination therapy with ifosfamide and doxorubicin, he was treated with only ifosfamide because he had minor arrhythmia and mild cardiac dysfunction. Ifosfamide ( $3.5 \mathrm{~g} \mathrm{x} 4$ days) was administered intravenously twice every 4 weeks. Platelet transfusion was given because thrombopenia occurred during the second cycle. There were no other complications. Third cycle chemotherapy with ifosfamide ( $3 \mathrm{~g} \mathrm{x} 4$ days) was started 80 days after final surgery and at 15 days after termination of radiotherapy. He felt shortness of breath since following administration. Dyspnea gradually increased, and chest X-ray and CT revealed ground-glass infiltrations in the right pulmonary lobe (Fig. 4). No evidence of infection was found. This condition was diagnosed as interstitial pneumonitis induced by ifosfamide and radiotherapy. Corticosteroid pulse therapy was started immediately. However, his oxygen concentration did not improve and the infiltrative lesion spread. He died due to respiratory failure despite ventilator support 110 days after the final surgery. No autopsy was performed.

\section{Discussion}

Malignant soft tissue tumors arising from the chest wall are rare. Excision with an adequate margin is usually performed and radiotherapy is not needed. However, it is often impossible to excise with a wide margin, such as in our case, after recurrence in the chest wall or inadequate excision because planning of the excision range is often difficult. The prognosis of malignant soft tissue tumors arising from the chest wall is poor compared with tumors arising from the extremities. Moreover, treatment of recurrent tumors is more difficult. Radiotherapy is additionally required in such difficult cases, 
and chemotherapy is performed to prevent local recurrence or metastases.

Our patient developed interstitial pneumonitis during chemotherapy after surgery and radiotherapy. Although chemotherapy is effective for tumor cells present throughout the body, antitumor drugs used in oncology may cause many adverse effects. One is interstitial pneumonitis. There are many reports of interstitial pneumonitis associated with chemoradiotherapy. The most common agents are methotrexate, cyclophosphamide, and bleomycin (2-4). Ifosfamide is one of the standard drugs for soft tissue sarcomas $(5,6)$. However, interstitial pneumonitis that is clearly associated with only ifosfamide, as in our case, has not been reported, although there have been some studies on interstitial pneumonitis after administration of combined chemotherapy including ifosfamide, which is used for bone and soft tissue sarcoma, and lymphoma (3,7-9).

Radiotherapy was considered as another cause of interstitial pneumonitis. Respiratory symptoms of this disease attributed to radiotherapy usually occur in the first 1-6 months following lung radiotherapy $(2,10)$. In our case, the onset of symptoms developed in three weeks, even though the irradiation field around the right subclavicular area was set not to include the lung as much as possible. Consequently, it unlikely that only radiotherapy caused interstitial pneumonitis. We think that combined chemotherapy and radiotherapy to the lung field may have caused this pneumonia. There are previous reports of radiation recall pneumonitis induced by chemotherapy after thoracic irradiation of malignant tumors such as lung cancer, lymphoma, rhabdomyosarcoma, and Ewing's sarcoma (11-13). Although there have been no previous reports, it was concluded that the pneumonitis was radiation recall pneumonitis which is a rare inflammatory reaction in the previously irradiated lung field due to administration of ifosfamide.

Some antitumor drugs are associated with a recall effect of pneumonitis after radiotherapy, especially doxorubicin (11,14), taxanes (14-16), gemcitabine (16), and molecular-target drugs such as vemurafenib (4) and erlotinib (10). The etiology and mechanism of radiation recall pneumonitis is unknown. The time interval from the end of radiation to interstitial pneumonia may not be very long $(15,16)$. The period to onset following radiotherapy was reported to be short in lung cancer and pediatric malignant disease, which are treated by combined chemotherapy and radiotherapy to the lung field $(4,8)$. The median time interval from beginning chemotherapy to radiation recall pneumonitis for lung cancer was $12 \mathrm{~h}$ for anthracyclines, 30 days for combination gemcitabine and docetaxel, and 59 days for taxanes (16). The time to pneumonitis was short in our case. To our knowledge, there have been no previous reports of interstitial pneumonitis associated with ifosfamide used as a single agent. Ifosfamide is a popular antitumor agent. It may be toxic and occasionally induce radiation recall, including pneumonitis.

Radiotherapy is usually given for residual tumors after incomplete excision or recurrent tumors for malignant bone and soft tissue tumors. Moreover, chemotherapy is performed to avoid local recurrence or to treat distant metastases. In this case, radiotherapy and chemotherapy were performed to avoid progression of residual tumors and to prevent distant metastases because this tumor was histologically highly malignant. Ifosfamide is a standard agents for malignant bone and soft tissue sarcomas, and may be used in such situations. We report a rare case of radiation recall pneumonia with rapid progress induced by only ifosfamide despite a small irradiated region. Ifosfamide following chest irradiation may cause enhanced toxicity. Interstitial pneumonitis, which is likely to become fatal, must be considered for sarcoma, especially that arising from the chest wall.

\section{References}

1. Burris HA III and Hurtig J: Radiation recall with anticancer agents. Oncologist 15: 1227-1237, 2010.

2. Shanholtz C: Acute life-threatening toxicity of cancer treatment Crit Care Clin 17: 483-502, 2001

3. Doi M, Okamoto Y, Yamauchi M, Naitou H and Shinozaki K: Bleomycin-induced pulmonary fibrosis after tumor lysis syndrome in a case of advanced yolk sac tumor treated with bleomycin, etoposide and cisplatin (BEP) chemotherapy. Int J Clin Oncol 17: 528-531, 2012

4. Forschner A,Zips D, Schraml C, Röcken M, Iordanou E, Leiter U, Weide B, Garbe C and Meier F: Radiation recall dermatitis and radiation pneumonitis during treatment with vemurafenib. Melanoma Res 24: 512-516, 2014.

5. Brunello A, Rizzato MD, Rastrelli M, Roma A, Maruzzo M, Basso U, Fiduccia P, Buzzaccarini MS, Scarzello G, Rossi CR, et al: Adjuvant chemotherapy for soft tissue sarcomas: A 10-year mono-institutional experience. J Cancer Res Clin Oncol 142: 679-685, 2016

6. Tanaka K, Mizusawa J, Fukuda H, Araki N, Chuman H, Takahashi M, Ozaki T, Hiruma T, Tsuchiya H, Morioka H, et al: Perioperative chemotherapy with ifosfamide and doxorubicin for high-grade soft tissue sarcomas in the extremities (JCOG0304). Jpn J Clin Oncol 45: 551-561, 2015.

7. Baker WJ, Fistel SJ, Jones RV and Weiss RB: Interstitial pneumonitis associated with ifosfamide therapy. Cancer 65: 2217-2221, 1990

8. Prestwich RJ, Picton SV, Glaser A and Taylor RE: Fatal pneumonitis in children with metastatic rhabdomyosarcoma following whole lung radiotherapy and sequential epirubicin. Pediatr Blood Cancer 48: 586-590, 2007.

9. Dincol D, Buyukcelik A, Dogan M, Akbulut H, Samur M, Demirkazik A, Senler FC, Onur H and Icli F: Long-term outcome of mesna, ifosfamide, mitoxantrone, etoposide (MINE) regimen as a consolidation in patients with aggressive non-Hodgkin lymphoma responding to CHOP. Med Oncol 27: 942-945, 2010.

10. Arakawa H, Johkoh T, Sakai F, Kusumoto M, Hataji O and Taguchi O: Exacerbation of radiation fibrosis with erlotinib: Another pattern of radiation recall phenomenon. Jpn J Radiol 29: 587-589, 2011.

11. Ma LD, Taylor GA, Wharam MD and Wiley JM: 'Recall' pneumonitis: Adriamycin potentiation of radiation pneumonitis in two children. Radiology 187: 465-467, 1993.

12. Minami-Shimmyo Y, Ohe Y, Yamamoto S, Sumi M, Nokihara $H$, Horinouchi H, Yamamoto N, Sekine I, Kubota K and Tamura T: Risk factors for treatment-related death associated with chemotherapy and thoracic radiotherapy for lung cancer. J Thorac Oncol 7: 177-182, 2012.

13. Fox AM, Dosoretz AP, Mauch PM, Chen YH, Fisher DC, LaCasce AS, Freedman AS, Silver B and Ng AK: Predictive factors for radiation pneumonitis in Hodgkin lymphoma patients receiving combined-modality therapy. Int J Radiat Oncol Biol Phys 83: 277-283, 2012.

14. Azria D, Magné N, Zouhair A, Castadot P, Culine S, Ychou M, Stupp R, Van Houtte P, Dubois JB and Ozsahin M: Radiation recall: A well recognized but neglected phenomenon. Cancer Treat Rev 31: 550-570, 2005.

15. Schweitzer VG, Juillard GJ, Bajada CL and Parker RG: Radiation recall dermatitis and pneumonitis in a patient treated with paclitaxel. Cancer 76: 1069-1072, 1995.

16. Ding X, Ji W, Li J, Zhang X and Wang L: Radiation recall pneumonitis induced by chemotherapy after thoracic radiotherapy for lung cancer. Radiat Oncol 6: 24, 2011. 